\title{
Una aplicación de experimentos de elección para identificar preferencias locales por opciones de conservación y desarrollo en el extremo sur de Chile
}

\author{
An application of choice experiments to identify local preferences for conservation and \\ development options at the extreme south of Chile
}

\author{
Claudia Cerda \\ Universidad de Chile, Facultad de Ciencias Forestales y Conservación de la Naturaleza, Departamento de Gestión Forestal y su \\ Medio Ambiente, Santa Rosa 11315, La Pintana, tel.: 9785903, clcerdaj@uchile.cl
}

\begin{abstract}
SUMMARY
This study uses a choice experiment (CE) to estimate economic values of environmental services that are not traded in markets, provided by forests of Navarino Island, Magallanes and Antártica Chilena, Chile. This is a pristine area with low level of economic development. It includes temperate forests of global interest to be preserved. However, development projects can threaten this condition. The central research question is: to know the economic preferences of the local community of Navarino for the environmental services provided by forests on the island. Scenarios with different levels of attributes and touristic development were analysed using a CE $(n=230)$. As most of the development options for Navarino include some loss of environmental services due to the pristine condition of the place, increases in income/year were offered as monetary compensation (willingness to accept). Participants' decisions about future development and conservation strategies were influenced by the following attributes: scenic beauty threatened by levels of tourism infrastructure, access restrictions due to both nature conservation and private interests, visits of the hummingbirds (Sephanoidessephanoides) and protection for an endemic moss (Tayloria mirabilis). Participants favoured a model of low impact development, represented by small-scale tourism. This research illustrates the application of a methodology to incorporate citizens preferences in conservation strategies.
\end{abstract}

Key words: choice experiment, willingness to accept, Navarino, economic valuation, preferences.

\section{RESUMEN}

Este estudio utiliza un experimento de elección (EE) para estimar valores económicos de servicios ambientales no transados en mercados, proporcionados por bosques de la isla Navarino, región de Magallanes y Antártica Chilena, Chile. Ésta es un área prístina con bajo nivel de desarrollo económico. Contiene bosques templados de interés mundial para ser conservados. Sin embargo, proyectos de desarrollo pueden amenazar esta condición. La pregunta central de investigación fue: conocer las preferencias económicas de la comunidad local de Navarino, por los servicios ambientales proporcionados por los bosques de la isla. Para ello se analizaron escenarios con diferentes niveles de atributos y de desarrollo turístico mediante un EE ( $\mathrm{n}=230)$. Como la mayoría de las opciones de desarrollo para Navarino incluyen alguna pérdida de servicios ambientales debido a la pristinidad del lugar, se ofrecieron incrementos en la renta anual como compensación monetaria (disposición a aceptar). Las decisiones de los participantes sobre futuras estrategias de desarrollo y conservación fueron influenciadas por los siguientes atributos: belleza del paisaje amenazada por niveles de infraestructura turística; restricciones de acceso a la naturaleza debido a intereses privados y de conservación, visitas de los picaflores (Sephanoides sephanoides) y protección para un musgo endémico (Tayloria mirabilis). Los participantes favorecieron un modelo de desarrollo de bajo impacto, representado por turismo a baja escala. Esta investigación ilustra la aplicación de una metodología que permite incorporar en las estrategias de conservación las preferencias de los ciudadanos.

Palabras clave: experimento de elección, disposición a aceptar, Navarino, valoración económica, preferencias.

\section{INTRODUCCIÓN}

Este estudio se genera ante la necesidad de mostrar cómo herramientas de valoración económica de servicios ambientales proporcionados por ecosistemas boscosos, que consideran preferencias locales, pueden contribuir con información para una mejor gestión de conflictos entre desarrollo y conservación de los sistemas naturales.

Los servicios ambientales corresponden a los benefi- cios que las personas obtienen desde los ecosistemas. Incluyen servicios de provisión como agua y alimentos, de regulación como control de inundaciones y desastres, de soporte como el ciclo de nutrientes, y culturales como los beneficios de la recreación, espirituales y religiosos (Millennium Ecosystem Assessment 2003). Cuando se hace referencia a preferencias locales, la valoración de los servicios ambientales se enfoca en aquellas personas que pueden ser directamente afectadas por decisiones políticas que 
afecten el estado actual de tales servicios. En el presente estudio esto se justifica en el hecho de que fue diseñado específicamente para contribuir como input a los procesos de participación local para definir acciones de conservación y desarrollo turístico en Navarino. Desde el punto de vista económico, los beneficios y costos de estas acciones son definidos en términos de preferencias económicas (Bateman et al. 2002). Para medir la magnitud del beneficio, se mide cuánto un individuo está dispuesto a sacrificar para obtenerlo. Contrariamente, para medir la magnitud del costo, se mide la compensación requerida por una persona para incurrir en él (Bateman et al. 2002).

Diferentes metodologías para aproximarse al valor económico de los servicios proporcionados por los ecosistemas han sido desarrolladas y aplicadas. Cuando no existen mercados para los servicios, las técnicas de preferencias declaradas permiten obtener valores económicos aproximados. Los experimentos de elección son métodos conocidos para este propósito.

Este estudio muestra una aplicación metodológica de un experimento de elección al problema de conflictos entre opciones de desarrollo y conservación que involucran cambios a servicios ambientales con relevancia local, proporcionados por ecosistemas boscosos de la isla Navarino, región de Magallanes, Chile. Se exploran preferencias económicas locales por cambios en diferentes servicios ambientales no transados en mercados, producto de la implementación de diferentes opciones de conservación y desarrollo potenciales futuras para la isla utilizando un formato de disposición a aceptar compensación (DAC). El énfasis de la valoración está dado en servicios de uso directo (aquellos actualmente usados por las personas, ya sea para propósitos comerciales o de recreación) y de no uso (en este caso, se refiere a los beneficios que genera a las personas la mera existencia de un recurso aunque nunca se haya hecho un uso directo del mismo y probablemente no se vaya a hacer en el futuro). Esto no quiere decir, sin embargo, que se considere que los servicios ambientales incorporados en el estudio tienen un único valor (uso o no uso). La distinción, en este caso, se hace con base en investigación cualitativa que indaga en el tipo de valor que los residentes de Navarino atribuyen a los servicios considerados (Barkmann et al. 2005). En este contexto, un recurso puede tener diferentes valores asociados, tanto de uso como de no uso. La separabilidad de los valores que las personas pueden atribuir a los servicios ambientales, ha sido planteada como una problemática compleja de solucionar en la valoración económica del ambiente natural (Loomis 1988). En este estudio, la clasificación de los servicios de acuerdo a las tipologías de valor se realiza considerando los argumentos de los participantes, aunque no se excluye la posibilidad de que exista incrustación de otros valores para los servicios específicos identificados.

La hipótesis central de este trabajo es que los habitantes de Navarino se comportan como consumidores sensitivos a cambios en la renta monetaria, en el contexto de la ges- tión de los bosques del lugar que afecta servicios ambientales relevantes para la comunidad. En función de ello, los objetivos planteados son: a) mostrar una aplicación metodológica de un experimento de elección para cuantificar valores económicos de servicios ambientales con énfasis en aquellos no transados en mercados convencionales y b) determinar si existen preferencias económicas de los habitantes de Navarino por servicios ambientales proporcionados por los bosques del lugar.

Parte de los datos empíricos de este estudio fueron también utilizados en la publicación de Cerda et al. (2007), en la cual se analiza la controversia conceptual en valoración económica relativa a las disparidades entre disposición a pagar y disposición a aceptar compensación. Para ello, en ese artículo se presentan dos estudios de caso, en Navarino e Indonesia, que utilizan experimentos de elección que obedecen a un diseño mixto del atributo monetario. Este artículo expande la aplicación de experimento de elección para conocer las preferencias de la población de la Isla Navarino con respecto a opciones de conservación y desarrollo que afectan servicios ambientales proporcionados por los bosques del lugar.

El uso de experimentos de elección (Hensher et al. 2005) da luces en la necesidad de contar con información sobre el valor económico de servicios ambientales que no cuentan con transacción en el mercado.

Los experimentos de elección se basan en la teoría de la utilidad aleatoria (McFadden 1973). Esta teoría propone que la utilidad de un bien se descompone en un componente observable que es función de un vector de atributos y características del individuo, y en un componente no observable conocido como error. Dado ciertos supuestos para la distribución del error, la probabilidad de elegir una opción se puede expresar como una distribución logística (McFadden 1973). Los atributos varían en distintos niveles los cuales se combinan para generar escenarios específicos (alternativas) que son seleccionados desde un universo de posibles escenarios (Hensher et al. 2005). Estos escenarios son presentados a los participantes en una serie de conjuntos de elecciones, donde cada conjunto contiene dos o más escenarios. Mediante la repetición de los conjuntos y una variación sistemática de los niveles de los atributos, el investigador puede inferir cuáles atributos influyen en la elección de un determinado escenario y evaluar transacciones entre atributos. Si se incorpora un atributo monetario, disposición a aceptar compensación (DAC) o disposición a pagar (DAP) pueden ser estimadas (Hanley et al. 1998). Si los cambios a los atributos representan pérdidas o deterioro respecto a la situación actual, se debe utilizar disposición a aceptar compensación (Freeman 1998). De lo contrario, si los escenarios representan en su totalidad mejoras respecto a la situación actual, el atributo monetario es diseñado como un costo adicional para el individuo a ser pagado cuando la alternativa sea implementada (DAP). Cuando las alternativas incluyen simultáneamente mejoras o deterioros a los atributos considerados producto de la 
implementación de diferentes acciones que pueden tener efectos negativos y positivos en los ecosistemas, diseños mixtos del vehículo de pago (disposición a aceptar compensación versus disposición a pagar) pueden ser utilizados (Hanley et al. 1998). Adicionalmente, los derechos de propiedad respecto al objeto de valoración también resulta ser un criterio esencial para la elección de la medida de valor a utilizar (Freeman 1998). Si las personas tienen la sensación de poseer los derechos de propiedad por un determinado servicio ambiental, se sugiere utilizar disposición a aceptar compensación.

El uso de experimentos de elección abre un interesante campo de investigación en Chile. Su aplicación puede estimular conciencia pública frente a la potencial pérdida de servicios ambientales debido al deterioro de ecosistemas boscosos (Barkmann et al. 2008). Ciudadanos y tomadores de decisión pueden entender mejor la contribución de los bosques al bienestar humano, y justificar recursos para asegurar que ellos sean utilizados sustentablemente. Esto es relevante, ya que la implementación y desarrollo exitoso de alternativas de uso y conservación pueden depender fuertemente de la aceptabilidad social de esas alternativas.

\section{MÉTODOS}

Navarino se ubica en el extremo sur de América, al norte del archipiélago Cabo de Hornos. Sus habitantes $(\mathrm{N}=2.000)$ se concentran en Puerto Williams. Sus ecosistemas forman parte de la ecorregión de bosques Subantárticos de Magallanes (Rozzi et al. 2004). Esta ecorregión presenta un mosaico de ecosistemas con características singulares y únicas a nivel mundial, libres de impacto humano directo y no están fragmentados. El interés por proyectos de turismo ha aumentado abruptamente en los últimos años, lo cual representa una amenaza potencial a la condición de pristinidad.

El estudio se llevó a cabo entre los años 2003-2006 y se enfocó esencialmente en servicios ambientales relevantes para la comunidad local proporcionados por los ecosistemas boscosos de la isla. Por servicios ambientales relevantes para la comunidad local se consideraron aquellas fuentes relevantes de beneficios de la naturaleza de Navarino mencionadas espontáneamente por los participantes del estudio (Cerda 2011). Para identificar estas fuentes, se analizaron ideas de la comunidad local de Navarino sobre servicios ambientales (Barkmann et al. 2005) a través de una fase previa de investigación cualitativa. Estas ideas fueron utilizadas para definir atributos a ser valorados utilizando un experimento de elección. Se utilizó el concepto de valor económico total (VET; Pearce y Moran 1994) para facilitar la clasificación de los atributos de acuerdo a las tipologías de valor expresada por los participantes. Los atributos fueron los siguientes: cambio en el paisaje por infraestructura turística (valor de uso directo), restricciones de acceso a la naturaleza debido a intereses privados y de conservación, posibilidad de observar animales y visitas de los picaflores (Sephanoides sephanoides (Lesson 1827) Familia: Trochilidae) con importancia cultural.

Adicionalmente, se incorporó como un atributo adicional la "probabilidad de extinción de una especie inconspicua”, específicamente un musgo endémico de la zona subantártica de Chile (Tayloria mirabilis (Cardot) Broth.). La inclusión de este atributo obedeció más bien a intereses de investigación relativos a evaluar el potencial de los experimentos de elección para resolver problemas metodológicos propios de la estimación económica de valores de existencia de especies, específicamente separación de valores, incrustación y motivaciones morales (Kahnemann y Knetsch 1992). En el contexto de la cuantificación económica de valores de existencia, empíricamente ha sido documentado que los consumidores y ciudadanos están dispuestos a sacrificar algo en orden de rescatar a una especie, por ejemplo, de su extinción, o para asegurar su existencia (Martín-López et al. 2008). Este es el caso, incluso si los individuos no tienen la intención de usar, alguna vez, la especie (Krutilla 1967). Actualmente se argumenta robustamente que la valoración económica de cambios ambientales que afectan la existencia de especies negativamente, "debe" considerar la pérdida potencial de los beneficios de la existencia de tal especie (Just y Hueth 2004).

Por la importancia mundial de la flora de musgos y líquenes de Magallanes (Rozzi et al. 2004) una especie de musgo endémica de los bosques subantárticos fue elegida para estos propósitos ${ }^{1}$. Los detalles metodológicos que permitieron aproximarse a evaluar el potencial de esta herramienta para resolver los problemas inherentes a la cuantificación de los beneficios económicos de la existencia de especies se encuentran en Cerda (2011).

Respecto al atributo “cambio en el paisaje por infraestructura turística”, inicialmente pretendió hacer referencia al daño estético al paisaje provocado por diferentes niveles de impacto de infraestructura turística. La fase cualitativa previa del estudio había revelado que los residentes otorgan gran importancia a los paisajes locales intactos. Para evitar una potencial manipulación del lenguaje, la redacción final del atributo y de sus explicaciones, se refirió solamente a "cambios al paisaje" producto de diferentes niveles de infraestructura turística.

El atributo fue operacionalizado a partir de cuatro niveles de variación: "cambio muy pequeño" correspondiente al statu quo, "cambio pequeño", "cambio mediano" y "gran cambio". Los cambios fueron representados por

${ }^{1}$ La diversidad de musgos y líquenes de Magallanes son de relevancia mundial (Rozzi et al. 2004). Los científicos presentes en la isla Navarino, a través de exitosos programas de educación y turismo sustentable enfocados a la comunidad de Puerto Williams, han hecho grandes esfuerzos por comunicar esta importancia. Incluso más, muchos residentes de Navarino han participado en programas de "turismo con lupa" cuyo propósito es dar a conocer la importancia de conservar las briófitas del lugar por su importancia mundial. De esta forma, aunque los beneficios de la existencia de musgos no aparecieron espontáneamente desde los participantes en la fase cualitativa del estudio, valorar la existencia de una especie específica de musgo no resulta en absoluto ajeno o poco relevante para los participantes. 
niveles progresivos de infraestructura turística, descritos por números y tipos de cabañas incluyendo un hotel para el "gran cambio", así como también senderos y caminos (figura 1). El nivel correspondiente al statu quo, fue representado por un "cambio muy pequeño" ya que infraestructura muy básica había sido construida en la isla hasta el momento del estudio, provocando un muy bajo impacto al paisaje. Era muy probable que se instalara nueva infraestructura ya que varios análisis habían mostrado un gran potencial de Navarino para el desarrollo del ecoturismo (Rozzi et al. 2004).

Respecto al vehículo de pago utilizado, el análisis de las medidas de bienestar para valorar cambios en los servicios ambientales incorporados en el estudio llevó a utilizar preferentemente un formato de disposición a aceptar compensación, el cual incorporó tres niveles, y un nivel de disposición a pagar, resultando finalmente en un diseño mixto. Los criterios que justifican este diseño del vehículo de pago se explican a continuación (Freeman 1998, Just y Hueth 2004).

Dirección de los cambios propuestos. El análisis de los cambios propuestos respecto a la situación actual en el escenario de valoración resulta esencial a la hora de decidir si disposición a aceptar compensación o disposición a pagar es la medida apropiada para valorar cambios ambientales. Si un proyecto específico daña la calidad ambiental, la medida adecuada es disposición a aceptar com- pensación. En el caso específico de Navarino, la situación ecológica de los ecosistemas es predominantemente prístina. Sin embargo, intereses económicos en áreas como turismo podrían afectar negativamente la biodiversidad de los bosques del lugar. Es probable que la mayoría de las opciones de desarrollo sustentable causen más de algún daño ambiental. Este análisis sugirió usar preferentemente un formato de disposición a aceptar compensación (Freeman 1998, Bateman et al. 2002, Just y Hueth 2004). Por otra parte, fue necesario considerar al mismo tiempo que procesos de planificación futuros también pueden resultar en mejoras ambientales, por ejemplo, con respecto a la preservación de bosques primarios de la isla y sus especies. Desde este punto de vista, una utilización exclusiva de disposición a aceptar compensación no reflejaría esta situación. En consecuencia, para el atributo monetario se utilizó un formato mixto con tres niveles de disposición a aceptar y un nivel de disposición a pagar.

Derechos de propiedad sobre el objeto de valoración. Ahlheim y Buchholz (2000) argumentan que si los participantes se sienten con derechos sobre la situación ambiental actual del territorio, es conveniente utilizar un formato de disposición a aceptar compensación. En este estudio, los deterioros ambientales fueron interpretados como una pérdida en la posición de los residentes de Navarino de que poseen derechos de propiedad sobre el territorio. Entrevistas cualitativas previas a la principal aplicación del

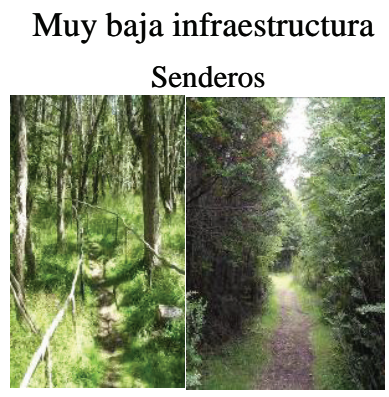

4 cabañas tipo A

\section{B aja infraestructura}

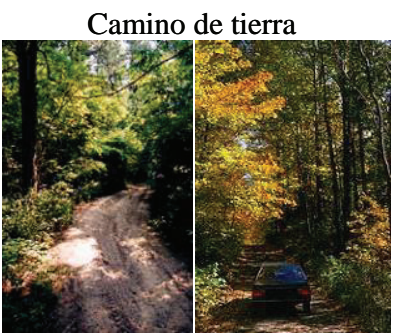

8 cabañas tipo A 6 cabañas tipo B
M ediana infraestructura

Camino de ripio

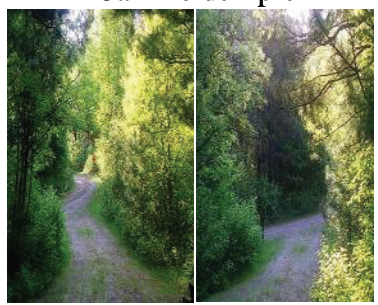

12 cabañas tipo A

12 cabañas tipo B

\section{A Ita infraestructura}

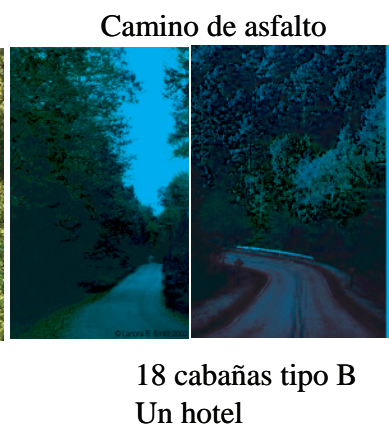

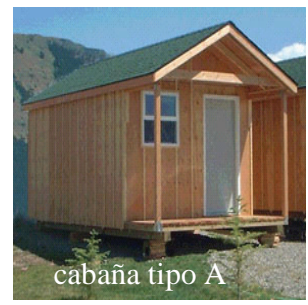
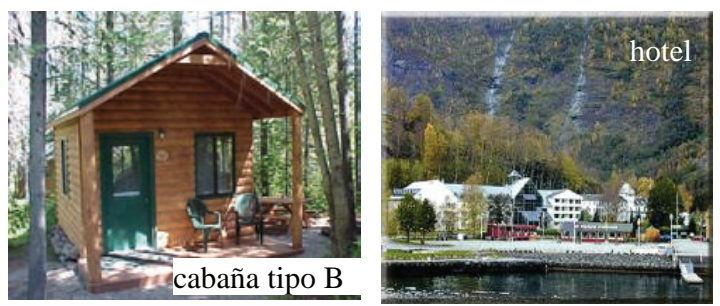

Figura 1. Imágenes utilizadas para presentar los niveles de cambio al paisaje por infraestructura turística a los participantes, durante la aplicación principal del experimento de elección. Progresión de los niveles: de izquierda a derecha. Los niveles fueron presentados al mismo tiempo, tal como se muestra en la figura.

Images used to present the levels of landscape change by tourism infrastructure to the participants, during the main application of the choice experiment. Progression of levels: from left to right. Levels were presented in the same order as shown in the figure. 
experimento de elección revelaron que los habitantes de la isla se sienten con ciertos derechos de calidad ambiental y de uso del territorio. De esta forma, infracciones a estos derechos, que los participantes podrían percibir desde las alternativas ofrecidas, sugirieron un formato mayoritario de disposición a aceptar compensación. En la fase cualitativa del estudio, el "acceso abierto" al paisaje y naturaleza de la isla fue a menudo referido como un aspecto positivo de vivir en Navarino. La visión de los residentes respecto a estos "derechos" llevó a los participantes a protestar en contra de la proposición de que ellos deberían pagar por mantener la actual naturaleza y paisajes de Navarino, incluyendo el espacio abierto para actividades de ocio y recreación.

Utilizando como marco de referencia el concepto de valor económico total (Pearce y Moran 1994), el cuadro 1 muestra los servicios ambientales valorados y sus niveles de variación.

Basado en el formato mixto mencionado, como atributo monetario se utilizó un "cambio en la renta/año" con los siguientes niveles de variación: -\$30.000, \$0 (statu quo), $+\$ 20.000,+\$ 30.000,+\$ 40.000$.

Los atributos y niveles del cuadro 1 y el atributo monetario, fueron combinados para construir escenarios de desarrollo a ser presentados a los participantes (cuadro 2). Estos escenarios fueron generados de acuerdo a un diseño experimental ortogonal de efectos principales (Louviere et al. 2000, Hensher et al. 2005). Para este diseño experimental se utilizó el software estadístico SPSS (15.0). Pos- teriormente, los escenarios fueron combinados en sets de elecciones con escenarios A y B y un statu quo utilizando procedimientos "mix and match" (Louviere et al. 2000). El diseño resultó en que cada persona debió enfrentarse a ocho conjuntos de elecciones, es decir, cada persona debió elegir ocho veces una opción dentro de cada conjunto.

Este procedimiento de diseño experimental permitió que se generaran escenarios probables de ocurrir, debido a que los atributos se plantearon de tal manera que la variación de uno no afectara a los otros. Por ejemplo, los cambios al paisaje y las restricciones de acceso fueron establecidos en lugares específicos de la isla diferentes a aquellos que los residentes usan activamente para recreación o donde tienen mayor posibilidad de observar diferentes animales. De esta forma, aquellas combinaciones que resultaron en escenarios donde por ejemplo se afectaba significativamente el paisaje, se restringía el acceso a la naturaleza y aun así se podían observar más animales que antes, resultan factibles ya que las personas pueden observar animales en lugares distintos y alejados de aquellos seleccionados para sufrir cambios al paisaje y restricciones de acceso. De esta forma, los escenarios reflejaron una gama de probables vías de desarrollo para la isla.

La estructura del cuestionario fue la siguiente: primero se planteó el objetivo del estudio. En una segunda sección los atributos y niveles fueron explicados exhaustivamente a los participantes. Se incorporaron preguntas abiertas específicas para cada atributo, con el objetivo de comprender mejor las visiones y conocimiento de los participantes

Cuadro 1. Servicios ambientales y niveles de variación. Environmental services and levels of variation.

\begin{tabular}{|c|c|c|c|}
\hline $\begin{array}{l}\text { Categoría en el valor } \\
\text { económico total }\end{array}$ & Dimensión de valor & Atributo (servicio) & Nivel de variación \\
\hline Uso directo & Belleza escénica & $\begin{array}{l}\text { Cambio en el paisaje por infraestructura } \\
\text { turística }\end{array}$ & $\begin{array}{l}\text { - Muy pequeño } \\
\text { - Pequeño } \\
\text { - Mediano } \\
\text { - Gran cambio }\end{array}$ \\
\hline Uso directo & $\begin{array}{l}\text { Acceso a la } \\
\text { naturaleza }\end{array}$ & $\begin{array}{l}\text { Restricciones de acceso a la naturaleza } \\
\text { debido a intereses privados o de } \\
\text { conservación }^{1}\end{array}$ & $\begin{array}{l}\text { - No restringido }{ }^{2} \\
\text { - Medianamente restringido } \\
\text { - Muy restringido }\end{array}$ \\
\hline Uso directo & $\begin{array}{l}\text { Estética a nivel de } \\
\text { especies }\end{array}$ & $\begin{array}{l}\text { Posibilidad de ver animales: carpinteros } \\
\text { (Campephilus magellanicus King), } \\
\text { guanacos (Lama guanicoe Müller), } \\
\text { cóndores (Vultur griphus Linnaeus) }\end{array}$ & $\begin{array}{l}\text { - } 25 \% \text { más que ahora } \\
\text { - Como ahora }{ }^{2} \\
\text { - } 25 \% \text { menos que ahora }\end{array}$ \\
\hline Uso directo & $\begin{array}{l}\text { Valor etno } \\
\text { simbólico }\end{array}$ & Visita de los picaflores & $\begin{array}{l}\text { - Ya no visitan Navarino } \\
\text { - Visitan pero no están protegidos² } \\
\text { - Visitan y están protegidos }\end{array}$ \\
\hline No uso & Valor de existencia & $\begin{array}{l}\text { Probabilidad de extinción de un musgo } \\
\text { endémico }\end{array}$ & $\begin{array}{l}\text { - Incrementada } \\
\text { - Baja }{ }^{2} \\
\text { - Muy baja }\end{array}$ \\
\hline
\end{tabular}

Muestra dividida: restricciones de acceso debido a intereses privados o para proyectos de conservación. ${ }^{2}$ Statu quo. 
Cuadro 2. Ejemplo de un conjunto de elecciones. Example of a set of choices.

\begin{tabular}{lccc}
\hline \multicolumn{1}{c}{ Atributo } & $\begin{array}{c}\text { Opción de desarrollo } \\
\text { A }\end{array}$ & $\begin{array}{c}\text { Opción de desarrollo } \\
\text { B }\end{array}$ & Statu quo \\
\hline $\begin{array}{l}\text { Cambio en el paisaje por infraestructura } \\
\text { turística }\end{array}$ & Cambio pequeño & Gran cambio & $\begin{array}{c}\text { Cambio muy } \\
\text { pequeño }\end{array}$ \\
\hline $\begin{array}{l}\text { Restricciones de acceso a la naturaleza debido } \\
\text { a intereses privados o de conservación }\end{array}$ & $\begin{array}{c}\text { Medianamente } \\
\text { restringido }\end{array}$ & Muy restringido & No restringido \\
\hline $\begin{array}{l}\text { Posibilidad de ver animales (carpinteros, } \\
\text { guanacos, cóndores) }\end{array}$ & Como ahora & $25 \%$ más que ahora & Como ahora \\
\hline $\begin{array}{l}\text { Visita de los picaflores } \\
\text { Probabilidad de extinción de un musgo } \\
\text { endémico }\end{array}$ & Ya no vienen & $\begin{array}{c}\text { Vienen pero no están } \\
\text { protegidos }\end{array}$ & $\begin{array}{c}\text { Vienen y están } \\
\text { protegidos }\end{array}$ \\
\hline Cambio en la renta/año & $+\$ 30.000$ & Baja & Muy baja \\
\hline
\end{tabular}

Yo elijo:

respecto a los objetos de valoración. Las preguntas se enfocaron en las motivaciones de los entrevistados para proteger los servicios ambientales seleccionados. La tercera parte del cuestionario incluyó los conjuntos de elecciones. Preguntas relativas a obtener información respecto a las características socioeconómicas de las personas fueron incorporadas en la última parte del instrumento.

La investigación se enfocó en la población permanente y económicamente activa de Navarino $(\mathrm{N}=1.328)$. Las bases del estudio establecían la necesidad de incorporar a todos los grupos de habitantes del lugar, lo cual llevó a la necesidad de estratificar la muestra. Para esto se utilizaron las categorías del Censo Nacional (INE 2002) ya que es la única fuente oficial de información poblacional existente para la localidad del estudio. Aunque profesores y personal de la armada fueron entrevistados utilizando un muestreo sistemático, sólo vía recomendaciones personales fue posible acceder a otros sectores relevantes de la población (pescadores, trabajadores en construcción), lo cual no hubiera sido factible de alcanzar incluso con largas campañas de terreno. De esta forma, para abarcar estos grupos fue necesario aplicar el muestreo de la "bola de nieve", con el objetivo de cubrir en forma balanceada a los grupos principales de residentes permanentes de acuerdo a categorías de ocupación del censo 2002 (INE 2002). Debido a que al utilizar este muestreo las personas pueden recomendar miembros de la población que conocen bien y que comparten sus mismas visiones, lo cual induciría a que pequeños grupos de interés no sean considerados, para minimizar sesgos se consultó a las personas a proporcionar información de miembros de la población que comparten sus mismas visiones y también a proporcionar información respecto a quienes no lo hacen (Lambert 1990), quienes también fueron entrevistados. Aunque este procedimiento resultó bastante satisfactorio pues fue posible cubrir va- riedad de miembros dentro de cada grupo ocupacional, se realizaron ajustes por sesgos de estratificación incorporando un factor de peso a cada participante (Greene 2007), de tal forma de corregir errores de muestreo con respecto a datos ocupacionales del último censo. Sin embargo, el muestreo no aleatorio utilizado impide cualquier pretensión de estricta representatividad.

La entrevista se llevó a cabo en forma personal por la autora de este artículo y dos estudiantes universitarios previamente entrenados para ello.

Finalmente, 230 residentes locales fueron entrevistados. Para el análisis econométrico de los datos, los niveles de los atributos ambientales fueron codificados utilizando variables dummies (Louviere et al. 2000, Bateman et al. 2002, Hensher et al. 2005). Modelos logísticos multinomiales (MLM; Hensher et al. 2005) fueron primeramente estimados para explicar la elección de los participantes por una alternativa de desarrollo, utilizando el software LIMDEP versión 3.0. Al aplicar la prueba de Hausman (Hensher et al. 2005), se determinó que los modelos estimados no cumplían con el axioma de independencia de alternativas irrelevantes ${ }^{2}$ (Louviere et al. 2000, Hensher et al. 2005) requerido para el modelo logístico multinomial. De esta forma, se usaron modelos logísticos anidados ${ }^{3}$, que parcialmente relajan la condición del axioma antes men${ }^{2}$ Este axioma establece que el radio de probabilidades de elegir una alternativa sobre otra (dado que ambas alternativas tienen una probabilidad de elección diferente de cero) no es afectado por la presencia o ausencia de cualquier alternativa adicional en el conjunto de elecciones (Louviere et al. 2000, p. 44). La prueba de Hausman es comúnmente utilizada para evaluar el supuesto de independencia de alternativas irrelevantes (Louviere et al. 2000, Hensher et al. 2005, Greene 2007). Para llevarla a cabo, los modelos logísticos multinomiales fueron reestimados sobre un subconjunto de alternativas.

${ }^{3}$ Ya que una descripción exhaustiva de este modelo va más allá del alcance de este artículo, se sugiere revisar a Hensher et al. (2005) y Louviere et al. (2000). 
cionado (Hensher et al. 2005). El modelo anidado es un modelo jerárquico de decisión que introduce una agrupación de alternativas; es diseñado para reconocer la posibilidad de que haya varianzas diferentes a través de las opciones y alguna correlación entre subconjuntos de opciones (Hensher et al. 2005). La presencia de estas posibilidades es equivalente a relajar el axioma de independencia de alternativas irrelevantes. Es conveniente dejar en claro que el modelo anidado no fue utilizado para aproximarse a un proceso de elección anidada (por ejemplo: primero decida si el statu quo es mejor que las alternativas que se ofrece, si no, elegir entre las alternativas que se ofrece), sino que fue utilizado para dar cuenta de diferentes variaciones sistemáticas de error entre las alternativas, lo cual puede llevar a violaciones del axioma de independencia de alternativas irrelevantes (Hensher et al. 20054).

La regla más importante para establecer la estructura jerárquica preferida es la maximización de la utilidad. Para probar esta condición, el valor inclusivo (VI) fue establecido en 1.0 (Hensher et al. 2005). Los parámetros de escala fueron normalizados al nivel más bajo (Hensher et al. 2005). Se seleccionó la mejor estructura de ajuste con un valor inclusivo entre 0 y 1 (Hensher et al. 2005). En el apéndice se muestra la estructura jerárquica del modelo. Esta estructura ha sido también utilizada por Barkmann et al. (2008).

Una alternativa específica constante (AEC) fue incorporada para capturar efectos del statu quo en las elecciones de los participantes (Hensher et al. 2005). Las pruebas de validación se ejecutaron mediante interacciones de los atributos con características socio-demográficas de los participantes (edad, género, renta, tiempo viviendo en la isla, educación).

Con los comandos requeridos por el software LIMDEP 3.0 para estimar el modelo anidado, se estimó una función de utilidad aditiva de la siguiente forma [1]:

$U=b_{1}{ }^{*}$ nivel2atributo $+b_{2 *}$ nivel3atributo ${ }_{1}+b_{3}$ nivel4atribu to $_{1}+\ldots+b_{6} *$ atributo $_{6}+b_{A E C} * A E C$

Donde:

$b_{1} \ldots b_{3}=$ coeficientes estimados de los niveles del atributo cambio en el paisaje por infraestructura turística con respecto al statu quo (nivel 1);

$b_{4} \ldots b_{6}=$ coeficientes de los otros atributos considerados, incluyendo el atributo cambio en la renta $b_{6}$;

$b_{a s c}=$ coeficiente estimado de la alternativa específica constante.

La significancia estadística de los coeficientes de los atributos ambientales y monetario se definió con $P<0,05$ (Bateman et al. 2002, Hensher et al. 2005), la cual fue utilizada para corroborar la hipótesis de la investigación. Si el cambio en la renta resulta ser estadísticamente significativo, quiere decir que los participantes se muestran como ${ }^{4}$ p. 418. consumidores sensitivos a cambios en la renta monetaria, en el contexto de la gestión de los bosques del lugar que afecta servicios ambientales relevantes para la comunidad.

Para la validación estadística del modelo global obtenido, se utilizó la prueba de razón de verosimilitud (Louviere et al. 2000, Hensher et al. 2005). Este procedimiento resulta ser adecuado para evaluar el ajuste global del modelo cuando el método de máxima verosimilitud es utilizado para estimar la utilidad de los parámetros de un modelo logístico (Louviere et al. 2000). Se usó el programa LIMDEP 3.0 que automáticamente realiza esta prueba, obteniendo la significancia estadística $(P)$ al nivel de aceptación de 0,05 . La significancia estadística se refiere a la probabilidad de que el estadístico Chi cuadrado sea mayor al valor de la prueba de razón de verosimilitud. Si $P$ es menor que el nivel de alfa, entonces se rechaza la hipótesis nula de que el modelo obtenido no es mejor que el modelo base de comparación (que incorpora sólo constantes).

Los valores económicos para los servicios considerados fueron calculados a partir de los coeficientes de los atributos estadísticamente significativos (Louviere et al. 2000, Hensher et al. 2005). El coeficiente del atributo monetario estimado, fue utilizado para calcular disposición a aceptar compensación marginal (Bateman et al. 2002, Hensher et al. 2005). La disposición a aceptar compensación marginal fue obtenida, entonces, dividiendo el coeficiente de cada atributo ambiental por el coeficiente del atributo monetario.

\section{RESULTADOS}

De los 230 participantes, 26 (11 \%) siempre eligieron el statu quo y 204 (89 \%) eligieron la opción A o B al menos una vez. Ningún participante manifestó dudas respecto a los cambios ofrecidos ni a los escenarios presentados. El modelo obtenido fue altamente significativo $\left(\chi^{2}, P<\right.$ 0,0001 ), por lo cual se rechaza la hipótesis nula de que este modelo no es mejor que el modelo base de comparación que incorpora sólo constantes. El estadístico valor inclusivo fue diferente de cero y menor que 1 , lo cual indica que el modelo es consistente con la maximización de la utilidad.

Los resultados del cuadro 3 muestran que los participantes fueron capaces de transar atributos ambientales por un atributo monetario. Con excepción del atributo "posibilidad de observar animales", todos influyeron en las decisiones de los participantes sobre alternativas de desarrollo $(P \leq 0,05)$. El atributo "cambio en la renta/año" resultó ser un predictor de elecciones significativo y afectó positivamente las funciones de utilidad de los participantes, como era esperado, ya que mayoritariamente un formato de disposición a aceptar compensación fue utilizado. El coeficiente de la alternativa específica constante fue negativo y significativo $(P<0,001)$, indicando una utilidad positiva asociada con alejarse del statu quo.

A partir de los coeficientes del cuadro 3, fue calculada la disposición a aceptar compensación marginal (cuadro 4). 
Cuadro 3. Modelo logístico anidado $(\mathrm{n}=230)$. Nested logit model $(n=230)$.

\begin{tabular}{lcc}
\hline \multicolumn{1}{c}{ Variable } & Coeficiente & Error estándar \\
\hline Cambio en el paisaje por infraestructura turística (pequeño cambio) & $0,1626^{* *}$ & 0,1138 \\
Cambio en el paisaje por infraestructura turística (mediano cambio) & $0,1407^{* * *}$ & 0,1157 \\
Cambio en el paisaje por infraestructura turística (gran cambio) & $-0,2608^{*}$ & 0,1170 \\
Restricciones de acceso a la naturaleza: & $0,1539^{* * *}$ & 0,0649 \\
$\quad$ - Por iniciativas económicas privadas & $0,1188^{* * *}$ & 0,0614 \\
$\quad$ - Por iniciativas de conservación & $0,0146^{\text {ns }}$ & 0,0020 \\
Posibilidad de observar carpinteros, guanacos y cóndores & $0,5552^{* * *}$ & 0,0519 \\
Visitas de los picaflores & $0,2153^{* * *}$ & 0,0516 \\
Protección para un musgo endémico & $0,0072^{* * *}$ & 0,0189 \\
Cambio en la renta (atributo monetario) & $-0,4125^{* * *}$ & 0,0830 \\
Constante (AEC) & $-1761,047$ & - \\
Log-likelihood & $<0,0001$ & - \\
$P\left(\right.$ Chi ${ }^{2}$ valor) & 0,97451 & - \\
Valor inclusivo $(\mathrm{VI})^{\#}$ & 0,1929 & - \\
Adj. $\rho^{2}$ (Pseudo-R $\left.{ }^{2}\right)$ & & - \\
\hline
\end{tabular}

${ }^{*} P \leq 0,05 ; * * P \leq 0,01 ; * * * P \leq 0,001 ;{ }^{\text {ns. }}$ : no significativo. " Valor no significativamente diferente de 1 , indicando que la condición de independencia de alternativas irrelevantes (Hensher et al. 2005) es válida para este modelo.

Cuadro 4. Disposición a aceptar compensación marginal. Marginal willingness to accept.

\begin{tabular}{lcc}
\hline \multicolumn{1}{c}{ Atributo } & $\begin{array}{c}\text { Unidad “marginal” utilizada para el cálculo de la } \\
\text { disposición a aceptar /codificación }\end{array}$ & $\begin{array}{c}\text { Disposición a } \\
\text { aceptar }\left(\$ \text { año }^{-1}\right)\end{array}$ \\
\hline $\begin{array}{l}\text { Cambio en el paisaje por infraestructura } \\
\text { turística }\end{array}$ & $\begin{array}{c}\text { Cambio muy pequeño a cambio pequeño } \\
\text { Cambio muy pequeño a cambio mediano } \\
\text { Cambio muy pequeño a gran cambio }\end{array}$ & $\begin{array}{c}22.580 \\
19.541 \\
\text { Restricciones de acceso: } \\
\quad \text { Actividades privadas }\end{array}$ \\
\hline \multicolumn{1}{c}{ Actividades de conservación } & $\begin{array}{c}\text { No implementar } \\
\text { Próximo nivel de restricción }\end{array}$ & 21.375 \\
\hline $\begin{array}{l}\text { Posibilidad de observar carpinteros, } \\
\text { guanacos y cóndores }\end{array}$ & Próximo nivel de restricción & 16.500 \\
\hline Los picaflores visitan la isla & - & - \\
\hline $\begin{array}{l}\text { Probabilidad de extinción de un musgo } \\
\text { endémico }\end{array}$ & Visitan /no protegidos a ya no vienen más a la isla \\
\hline AEC & $\begin{array}{c}\text { Baja } \\
\text { a incrementada }\end{array}$ & 77.111 \\
\hline
\end{tabular}

${ }^{1}$ El paso marginal representa un cambio desde el nivel de status quo a un nivel adyacente del atributo.

Para el caso del atributo cambio en el paisaje por infraestructura turística, por pasar del statu quo "cambio muy pequeño" a un "gran cambio", representado por caminos pavimentados y un gran hotel, la mínima compensación requerida es $\$ 36.220$ por año. El signo negativo de la disposición a aceptar compensación, indica un efecto negativo en el bienestar asociado a este cambio.

$\mathrm{Si}$ "pequeños cambios al paisaje" representados por más cabañas turísticas y mejores senderos no son implementados, la disposición a aceptar compensación es de aproximadamente $\$ 22.500$ por persona y por año, por renunciar al cambio. La disposición a aceptar compensación resultó un poco menor si no se implementan "cambios medianos".

Si no se implementan restricciones de acceso a la naturaleza de la isla, los residentes requieren una compensación de $\$ 21.375$ por año, cuando esas restricciones son impuestas por actividades privadas, y de \$ 16.500 cuando se trata de restricciones por conservación.

Si los picaflores dejan de visitar Navarino, la compensación requerida es $\$ 77.111$ por persona por año.

Un incremento en la probabilidad de extinción de 
un musgo endémico de los bosques subantárticos desde "baja" a "incrementada" requiere una compensación de $\$ 30.000$ por año.

Los participantes, en promedio, tienen un sesgo del statu quo equivalente a aproximadamente $\$ 57.000$ por año. El signo negativo indica una utilidad negativa asociada al statu quo.

\section{DISCUSIÓN}

La significancia del atributo monetario indica que los residentes locales se comportan como consumidores sensitivos a cambios en la renta en el contexto de la gestión de servicios ambientales, lo cual permite aceptar la hipótesis central de la investigación.

El modelo econométrico muestra que los residentes están dispuestos a aceptar cambios en el paisaje por infraestructura turística. Sin embargo, un análisis detallado de los coeficientes de cada nivel de variación del atributo, muestra una utilidad negativa asociada con el último nivel de variación representado por un gran cambio, producto de la instalación de grandes hoteles y caminos pavimentados (cuadros 3 y 4). De esta forma, los residentes prefieren un desarrollo de turismo de bajo impacto. Aceptan cambios a la belleza escénica en favor de infraestructura turística adicional, pero hasta un cierto límite. Esto indica que, no sólo se percibe el daño a la belleza escénica, sino que también beneficios económicos que infraestructura turística adicional podría traer a la isla.

Las restricciones de acceso para iniciativas privadas y para conservación, son positivamente vistas por los habitantes. Probablemente los participantes asociaron las iniciativas privadas con el beneficio económico que el turismo podría traer al lugar. Al momento de la entrevista este escenario era bastante probable ya que se habían garantizado concesiones para actividades turísticas.

Los picaflores son muy valorados, lo cual indica una gran apreciación de la cultura Yagán. El estudio muestra también evidencia respecto al valor económico de la conservación de especies inconspicuas, representadas, en este caso, por un musgo endémico. En el modelo econométrico obtenido, el coeficiente de este atributo fue altamente significativo. Los participantes efectivamente transaron mayores riesgos de extinción por beneficios económicos, enfrentando transacciones entre conservación, desarrollo socioeconómico y cambios en la renta. Respecto a este atributo, los programas de educación ambiental y turismo sustentable en Navarino que están enfocados a destacar la importancia mundial de las briófitas de la región, permitieron que, aunque este atributo no apareciera espontáneamente en la fase cualitativa del estudio, no resultara ajeno o poco familiar a los entrevistados. Aún si hubiera sido el caso de que los participantes no supieran absolutamente nada de la existencia de la especie de musgo endémico en cuestión y de esta forma no ganan utilidad desde el conocimiento de que esta especie reside en Navarino, la entre- vista de valoración, representa un punto de partida en la generación de preferencias (Kopp 1992, Fisher y Hanley 2007). Si las personas tienen la convicción de que los seres humanos deben preservar y respetar el mundo natural es muy probable que ellos sufrirán pérdida de bienestar al enterarse de que una especie endémica del lugar donde viven puede extinguirse por acciones antrópicas. Las personas no necesitan conocer a las especies por su nombre, o tenerlas plenamente identificadas para sufrir pérdidas de bienestar al enterarse de sus problemas de conservación (Kopp 1992). La estimación cuantitativa de valores existencia es, sin embargo, un procedimiento complejo, y requiere adaptación de estrategias específicas de valoración. Algunos avances metodológicos pueden ser vistos al usar experimentos de elección: al ofrecer transacciones más realistas de conservación-desarrollo e incluir varios valores simultáneamente lo cual mejora la separación de valores a través del requerimiento de que los participantes transen entre diferentes categorías de valor. También debieran incorporarse en la entrevista de valoración preguntas abiertas que den a los participantes la oportunidad de explicar sus elecciones.

Un resultado inesperado del estudio es la indiferencia manifestada por los entrevistados frente a la posibilidad de observar carpinteros, guanacos y cóndores. El carpintero es una especie que durante la investigación estaba siendo estudiada por varios científicos de otras regiones del mundo. Ha sido propuesto como especie emblemática para la conservación de los ecosistemas de Navarino. Previo a este estudio, los habitantes de la isla habían respondido numerosas encuestas para identificar el conocimiento local sobre esta especie. Resulta muy familiar para los residentes locales. De esta forma, el efecto de la información, pudo haber afectado la valoración incrustando a cóndores y guanacos. Al momento de la entrevista, muchos entrevistados manifestaron que les gustaría ver más esfuerzos de investigación hacia otras especies diferentes del carpintero.

Se obtuvieron valores monetarios entre \$ 16.000 y $\$ 78.000$ por año y por persona para cambios marginales a los atributos considerados. Como mayoritariamente se usaron incrementos en la renta como atributo monetario, estos valores son interpretados como la mínima disposición a aceptar por renunciar/implementar los cambios ofrecidos.

En este contexto, Cerda et al. (2007) presentan análisis econométricos que revelaron un radio de disposición a aceptar/disposición a pagar de 2,8. Esto quiere decir que la valoración promedio es marcadamente más baja si se les pregunta a los participantes por su disposición a pagar por evitar/implementar los cambios ofrecidos. Este valor es cercano al radio de 3,2 encontrado en un meta análisis de un estudio de valoración económica de Sayman y Önçüler (2005). Como la mayoría de los estudios de valoración económica utilizan un formato de disposición a pagar, el radio de 2,8 puede ser utilizado para po- 
der comparar los valores obtenidos con los resultantes de otros estudios. De esta forma, una disposición al pago de entre $\$ 5.000$ a $\$ 24.000$ año por persona es obtenida, lo cual equivale aproximadamente a porcentajes de la renta de los residentes de Navarino de entre 1 y $5 \%$. Otros estudios reportan valores de disposición a pagar de entre 0,1 y $1 \%$ de la renta de los participantes (Hynes y Hanley 2009). Sin embargo, los concretos lineamientos de planificación en Navarino, la situación ecológica del área y la sensación que tienen los habitantes respecto sus derechos sobre el territorio, permiten argumentar por la utilización de los resultados del estudio en términos de disposición a aceptar compensación. Este trabajo puede contribuir como un estudio de caso sobre cómo diseñar estrategias de uso y conservación de los bosques chilenos apoyadas por ciudadanos que se benefician directamente de ellos. La metodología utilizada constituyó una herramienta que permitió a los residentes expresar su "voz económica" con respecto a diferentes alternativas de sustentabilidad en Navarino.

\section{CONCLUSIONES}

La aplicación de experimentos de elección al problema de conflictos entre desarrollo y conservación de una de las regiones más prístinas del mundo reveló que los habitantes fueron capaces de transar entre conservación y desarrollo turístico. Mientras el actual uso de la tierra de Navarino, específicamente el plan seccional, permite proyectos turísticos a gran escala, los residentes locales favorecen un modelo de desarrollo turístico a pequeña escala. Iniciativas privadas de conservación son valoradas incluso si ellas resultan en restricciones de acceso para la realización de actividades recreativas. Los habitantes de isla Navarino valoran el patrimonio natural y cultural del lugar estando dispuestos a sacrificar ingreso para la protección de animales y plantas de los bosques, especialmente los picaflores.

\section{AGRADECIMIENTOS}

Al Ministerio de Educación e Investigación Alemán por el financiamiento.

\section{REFERENCIAS}

Ahlheim M, W Buchholz. 2000. WTP or WTA - Is that the question? Reflections on the differences between "willingness to pay" and "willingness to accept”. Zeitschrift für Umweltpolitik und Umweltrecht 23: 253-272.

Barkmann J, C Cerda, R Marggraf. 2005. Interdisziplinäre Analyse von Naturbildern: Notwendige Voraussetzung für die ökonomische Bewertung der natürlichen Umwelt. Umweltpsychologie 9: 10-28.

Barkmann J, K Glenk, A Keil, C Leemhuis, N Dietrich, G Gerold, R Marggraf. 2008. Confronting unfamiliarity with ecosystem functions: the case for an ecosystem service approach to environmental valuation with stated preference methods. Ecological Economics 65: 48-62.
Bateman I, R Carson, B Day, M Hanemann, N Hanley, T Hett, M Jones-Lee, G Loomes, S Mourato, E Özdemiroglu, OBE DW Pearce, R Sugden, R Swanson. 2002. Economic valuation with stated preference techniques: A manual. Cheltenham, UK. Edward Elgar. 580 p.

Cerda C, I Diafas, J Barkmann, J Mburu, R Marggraf. 2007. WTP or WTA, or both? Experiences from two choice experiments for early planning stages. In Meyerhoff J, N Lienhoff, P Elsasser eds. Stated preference methods for environmental valuation: applications from Austria and Germany. Marburg, Germany. Metropolis Verlag. p. 139-173.

Cerda C. 2011. Valuing biological diversity in Chile. Mauritius. VDM Verlag. 190 p.

Fischer A, N Hanley. 2007. Analysing decision behaviour in stated preference surveys: a consumer psychological approach. Ecological Economics 61: 303-314.

Freeman AM. 1998. The economic approach to environmental policy: the selected essays of A. Myrick Freeman III. Cheltenham, UK. Edwar Elgar. 516 p.

Greene W. 2007. NLogit Version 4.0. Reference Guide. Australia. Econometric Software. 300 p.

Hanley N, R Wright, W Adamowicz. 1998. Contingent Valuation versus Choice Experiments: Estimating the Benefits of Environmentally Sensitive Areas in Scotland. Journal of Agricultural Economics 49: 1-15.

Hensher D, J Rose, W Greene. 2005. Applied choice methods A Primer. Cambridge, UK. Cambridge University Press. $742 \mathrm{p}$.

Hynes S, N Hanley. 2009. The "Crex-crex" lament: estimating landowners willingness to pay for corncrake conservation on Irish farmland. Biological Conservation 142: 180-188.

INE (Instituto Nacional de Estadísticas, CL). 2002. Censo Nacional de Población. Consultado 21 ene. 2006. Disponible en http://www.ine.cl

Just RE, DL Hueth. 2004. The Welfare Economics of Public Policy - A Practical Approach to Project and Policy Evaluation. Cheltenham, UK. Edward Elgar. 687 p.

Kahneman D, JL Knetsch. 1992. Valuing public goods: the purchase of moral satisfaction. Journal of Environmental Economics and Management 22: 57-70.

Krutilla J. 1967. Conservation Reconsidered. American Economic Review 57: 777-786.

Kopp R. 1992. Why existence value should be included in costbenefit analysis. In Weimer DL ed. Cost-Benefit Analysis and Public Policy. Oxford, UK. Blackwell Publishing . DOI: $10.1002 / 9781444307177 . c h 19$.

Lambert E. 1990. The collection and interpretation of data from hidden populations. NIDA Research Monograph. Washington DC, USA. US Government Printing Office. 162 p.

Loomis J. (1988). Broadening the Concept and Measurement of Existence Value. Journal of Agricultural and Resource Economics 17(1): 23-29.

Louviere J, DA Hensher, JD Swait. 2000. Stated choice methods - analysis and application. Cambridge, UK. Cambridge University Press. 95 p.

McFadden D. 1973. Conditional logit analysis of qualitative choice behaviour. In Zarembka P ed. Frontiers in Econometric. New York, USA. Academic Press. p. 105-142.

Martín-López B, C Montes, J Benayas. 2008. Economic Valuation of Biodiversity Conservation: the Meaning of Numbers. Conservation Biology 22: 624-635.

Millennium Ecosystem Assessment. 2003. Ecosystems and hu- 
man well-being. A framework for assessment. Washington, USA. Island Press. 245 p.

Pearce D, D Moran. 1994. The economic value of biodiversity. London, UK. Earthscan. 172 p.

Rozzi R, F Massardo, C Anderson. 2004. Reserva de biósfera Cabo de Hornos. Una propuesta de conservación y turismo para el desarrollo sustentable en el extremo austral de América. Punta Arenas, Chile. Ediciones de la Universidad de Magallanes. 263 p.

Sayman S, A Önçüler. 2005. Effects of study design characteristics on the WTA-WTP disparity: A meta analytical framework. Journal of Economic Psychology 26: 289-312.

Recibido: 04.04.11

Aceptado: 16.08.11 
